\title{
A Stochastic Optimal Control Of Dc Pension Fund Driven By Fractional Brownian Motion With Hurst Parameter $>\alpha=\mathbf{2}^{-\mathbf{1}}$
}

\section{Onwukwe Ijioma}

\begin{abstract}
The problem of A fund manager is to minimize the expected utility loss function, the noise generated in the dynamics of the wealth process are driven by fractional Brownian motions with long range dependence (if $\mathrm{H}>1 / 2$ ).

We replaced the classical Brownian motion by fractional Brownian motion with Hurst parameter more than 1/2 . We finally use time-inversion of diffusions to obtain singular equations.
\end{abstract}

Index Terms - Defined- Contribution, Fractional Brownian motion, Stochastic optimal control. 2000 MSC: 60H15, 62H14.

\section{INTRODUCTION}

In a defined contribution (DC) pension scheme, the financial risk is the responsibility of the participating members. Contributions are prefixed; the benefits are dependent on the performance of the respective portfolios during the active periods of the members. Arising from the demographic evolution and the rapid development of the equity market, DC schemes have become global in the pension market.

The successful implementation of this scheme is due to the investment strategy during the accumulation phase in the DC scheme.

There are two approaches to the examination of the investment strategies as pioneered by [8]

The second approach called the Martingale method was developed by [2].

The novel approach in this paper is to investigate the structure of the noises generated in the dynamics of wealth processes to fractional Brownian motion (fBm) with long range dependence in the pension fund.

We recast the usual classical tool of optimal control optimization problem into a linear stochastic differential equation (SDE) driven by $\mathrm{fBm}$ with Hurst parameter $\mathrm{H}>1 / 2$ and under time inversion of certain diffusions and obtained some singular equations.

The rest of the work is organized as follows. In section 2, we introduced the classical model related to the problem of the pension fund management. In section 3 , we considered the concept of fractional Brownian motion for our work with Hurst parameter $\mathrm{H}>1 / 2$ for long range dependence. In section 4 , we give problem formulation and finally in section 5 , we use the time-inversion of certain diffusion to derive the solution of a singular equation.

Onwukwe Ijioma, Department of Mathematics, Abia State University, Uturu, Nigeria

\section{CLASSICAL PENSION FUND ROCESS:}

Merton [9] pioneered most of the pension fund models. Consider a market structure with two financial assets.

The riskless asset is governed by the dynamics, whose price at time $t$ is denoted by

$$
\begin{aligned}
& S_{0}(t),(t>0) \text { is } \\
& d S_{0}(t)=r S_{0}(t)
\end{aligned}
$$

where $r$ is the rate of interest.

The risky asset (stock) whose price at time $t$, denoted by $S_{1}(t)$ is governed by the dynamics defined by $d S_{1}(t)=S_{1}(t)(\mu d t+\sigma d w(t)$

where $\mu$ and $\sigma$ are parameters explaining the expected instantaneous rate of return of the risky asset and volatility of respectively. $W(t)$ is a normalized Gaussian white noise with zero mean and unit variance.

Given a fixed contribution rate $\lambda$, the invested fund proportion in risky asset at time $t$ is given by $y(t)$ and the remaining proportion $(1-y(t))$ is invested into the riskless asset.

The wealth process $x(t)$ at time $t$ is governed by the SDE $d x(t)=[y(t) x(t)+(1-y(t)) r x(t)+c] d t+$ $x(t) \sigma d w(t)$

(3) where $x(0)=x_{0}$.

The aim of the pension fund management is to maximize the expected utility at the exit from the scheme at time $T$.

Under some suitable conditions of (3), the optimal control problem is formulated as :

$\max _{y(t)} E(u(x(T)))$

The HJB yield the result

$$
\begin{aligned}
& \max _{y}\left\{v_{t}+[y(t)(\mu-r) x+r x] v_{x}+\right. \\
& \left.\frac{1}{2} y(t)^{2} \sigma^{2} x^{2} v_{x x}\right\}=0
\end{aligned}
$$

Where $v(t, x)$ is the value function, $v_{t}, v_{x}, v_{x x}$ respectively denote the partial derivatives

with respect to time and wealth.

The first order maximizing conditions for the optimal strategy $y^{*}(t)$ is

$$
\begin{aligned}
& y^{*}(t)=\frac{(y-\mu) v_{x}}{\sigma^{2} x v_{x x}} \\
& \mu(x(\mu T))=\frac{x^{y}(\tau)}{y}, y \in(-\infty, 1) \text { and }
\end{aligned}
$$


$y^{*}(t)=\frac{\mu-\gamma}{\sigma^{2}} \cdot \frac{1}{(1-p)}$

The prevailing government policies make some noises not to be Gaussian white

noises, but fractional white noises with short range dependence.

\section{FRACTIONAL BROWNIAN MOTION:}

Fractional Brownian motion ( $\mathrm{fBm}$ ) with Hurst parameter $H \in(0,1)$ is a zero mean Gaussian process $B^{H}=\left\{B_{t}^{H}, t \geq 0\right\}$ with covariance function:

$R_{H}(s, t)=\frac{1}{2}\left(t^{2 H}+S^{2 H}-\mid t-\right.$

$\left.\left.s\right|^{2 H}\right)$

The (fBm) can be constructed from the classical Brownian motion which was proposed by Mandelbrot and Van Ness (1968), where a stochastic integral representation in terms of a standard Brownian motion was obtained.

The self-similar and long range dependence (if $H>\frac{1}{2}$ ) as proposed in this work and the properties of $\mathrm{fBm}$ make this process a useful driving noise in models arising in physics, telecommunication networks, finance and other fields.

We define self-similarity of a process $X=\left(X_{t}, t \in(-\infty, \infty)\right)$ by means of its distribution. If $\left(X_{a t}\right)$ and $a^{H}\left(X_{y}\right)$ have identical finite-dimensional distributions for all $>0$, then $X$ is self-similar with parameter $H$.

Definition 1: A process $X$ is called (exactly) second-order self-similar with self-similarity parameter $H=1-\frac{\beta}{2}$, if for all $m=1,2, \ldots$,

$\operatorname{Var}\left[X^{(m)}\right]=\sigma^{2} m^{-\beta}$

And

$r^{(m)}(k)=r(k)=\frac{1}{2}\left((k+1)^{2 H}-2 k^{2 H}+\right.$

$\left.|k-1|^{2 H}\right), k \geq 0$

(9) where $r^{(m)}$ is the autocorrelation function of $X^{(m)}$

Definition 2: A process $X$ is called (asymptotically) second-order self-similar with self-similarity parameter $H=1-\frac{\beta}{2}$ if for all large $k$, then $r^{(m)}(k) \rightarrow r(k)$ as $m \rightarrow \infty$.

From $B^{H}$ is not a semi martingale and is not a Markov process if $H \neq \frac{1}{2}$ (see Rogers, L.C.G.(1997), this means that the usual stochastic calculus is not applicable for $\left(B^{H}, t \geq 0\right)$, if $H \in\left(\frac{1}{2}, 1\right)$.

Robust research has been done in the recent years on new techniques to define stochastic integrals with respect to $\mathrm{fBm}$.
Our interest is when $H>\frac{1}{2}$, then one can use a path-wise approach to define integrals with respect to the $\mathrm{fBm}$ (see Young, L.C.(1936)).

Alternatively, the approach to define path-wise integrals with respect to a $\mathrm{fBm}$ when $H>\frac{1}{2}$ is based on fractional calculus as introduced by Feyel and Del la Pradelle(1996).

We are interested in the structure and properties of one-dimensional linear stochastic differential equation defined as:

$$
\begin{gathered}
X_{t}=B_{t}^{H}+\int_{0}^{t} X_{u} d \mu(u), 0 \leq t \leq 1 \\
\text { where } B_{t}^{H}
\end{gathered}
$$

Brownian motion with Hurst parameter $H \in\left(\frac{1}{2}, 1\right)$.

\section{PROBLEM FORMULATION:}

Let $\Omega=C_{0}\left(R^{+}, R\right)$ be the Frechet space of real-valued continuous functions on $\mathrm{R}^{+}$with the initial value zero and topology of local uniform convergence.

For a fixed Hurst constant $H_{3} \frac{1}{2}<H<1$.

Define

$\Phi(s, t)=H(2 H-1)|s-t|^{2 H-2} ; s, t \in$

R (11)

\section{Let $f: \mathbb{R} \rightarrow R$ be measurable}

Then $f \in L_{\Phi}^{2}(\mathbb{R})$ if $|f|_{\Phi}^{2}=$

$\iint_{R, R} f(s) f(t) \Phi(s, t) d s d t<\infty$

Let $L_{\Phi}^{2}(R)$ be equipped with the inner product

$(f . g)_{\Phi}=\iint_{R R} f(s) g(t) \Phi(s, t) d s d t$

$f, g \in L_{\Phi}^{2}(R)$

Then $L_{\Phi}^{2}(R)$ becomes a separable Hilbert space with a probability measure $P^{H}$ on $(\Omega, \mathcal{F})$ where $\mathcal{F}$ is the usual

Borel $\sigma$-algebra on $\Omega$, the process is a fractional

Brownian motion (fBm),

By direct computation of (11)

$$
\begin{aligned}
& E\left[B^{H}(t) B^{H}(s)\right]= \\
& \iint_{R R} \Phi_{H}(u-v) d u d v
\end{aligned}
$$

Let $\left(X_{t}, t \geq 0\right.$ be the Gaussian process having the solution of

$$
X_{t}=B_{t}^{H}+\int_{0}^{t} X_{u} d \mu(\mu), 0 \leq t \leq 1(16)
$$

where $\mu$ is a Radon diffuse measure on $[0,1]$

By Bochner-Minlos theorem there exist a probability measure $\mu_{\oplus}$ such that 
$\int_{\Omega} \exp \left(i\langle\omega, f\rangle d \mu_{\Phi}(\omega)=e^{-\frac{1}{2}|f|^{2}}\right.$ or all $f \in S(R)$

It follows from (17) that

$\mathbb{E}_{\mu_{\Phi}}\left[\left\langle v_{v} f\right\rangle\right]=0$ and $\mathbb{E}_{\mu_{\Phi}}\left[\left\langle x_{s} f\right\rangle^{2}\right]=|f|^{2}$

We can redefine

$\hat{B}_{H}(t)=\hat{B}_{H}(t, \omega)=\langle\omega\rangle=,\left\langle\omega, X_{[0, t]}().\right\rangle$ as an element

of an $L^{2}\left(\mu_{\Phi}\right)$ for each $t \in R$, by Kolmogorov's theorem

$\hat{B}_{H}(t)$ has a $t$-continuous denoted by $B_{H}(t)$.

From (18), it is clear that $B_{H}(t)$ is a Gaussian process with $\mathbb{E}_{\mu_{\phi}}\left[B_{H}(t)\right]=0$ and $\mathbb{E}_{\mu_{\phi}}\left[B_{H}(s) B_{H}(T)\right]=$ $\frac{1}{2}\left\{|t|^{2 H}+|s|^{2 H}-|t-s|^{2 H}\right\}$

It follows that $B_{H}(t)$ is a fractional Brownian motion.

\section{TIME-INVERSION OF DIFFUSION PROCESS.}

We consider the following singular SDE: $X_{\mathrm{t}}=x+B_{\mathrm{t}}^{H}+\int_{0}^{t} b\left(u, X_{\mathrm{u}}\right) d u, t \geq 0(20)$

where the function $b(s, x)$ has a singularity at $s=0$.

We now establish the connection to some diffusions $\left(X_{t}, t \geq 0\right)$ which is a solution to (20) as

$X_{t}=B_{t}^{H}+2 H \int_{0}^{t} \frac{X_{s}}{s} d s, t \geq 0$

using time-inversion.

Let the process $\left(X_{t}\right)$ be a adapted to the filtration of $\left(B_{t}^{H}\right)$ and (20) one strong solution. By special choice of $s \in(0, t)$, we have

$\frac{X_{t}}{t^{2 H}}=\frac{X_{s}}{s^{2 H}}-2 H \int_{s}^{t} \frac{X_{u}}{u^{2 H+1}} d u+\int_{s}^{t} b \frac{\left(u, X_{u}\right)}{u^{2 H}} d u+\int_{s}^{t} \frac{d B_{u}^{H}}{u^{2 H}}$

$\lim _{t \rightarrow+\infty} \frac{X_{t}}{t^{2 H}} \rightarrow 0$ and $\lim _{t \rightarrow+\infty} \int_{s}^{t} \frac{X_{u}}{u^{2 H+1}} d u \quad$ exist a.s.

(23) using (22) and (23), it follows that

$$
\frac{X_{s}}{s^{2 H}}-2 H \int_{s}^{\infty} \frac{X_{u}}{u^{2 H+1}} d u+\int_{s}^{\infty} \frac{b\left(u, X_{u}\right)}{u^{2 H}} d u+\int_{s}^{\infty} \frac{d B_{u}^{H}}{u^{2 H}}=0
$$

and $\beta_{t}^{H}=-\int_{1 / t}^{\infty} \frac{d B_{u}^{H}}{u^{2 H}}, t>0 \quad$ is a $\mathrm{fBm}$.

Definition 4: Let $(S)_{H}$ be the set of all $\psi(\omega)=\sum_{\alpha \in I} a_{\alpha} H_{\alpha}(\omega) \in L^{2}\left(\mu_{\phi}\right)$ such that

$$
\|\psi\|_{H, k}^{2}=\sum_{\alpha \in I} \alpha ! a_{\alpha}^{2}(2 N)^{k \alpha}<\infty \quad \forall k \in N
$$

where $(2 N)^{\gamma}=\prod_{j}\left(2_{j}\right)^{\gamma j} \quad$ if $\quad \gamma=\left(\gamma_{1}, \ldots, \gamma_{m}\right) \in I$

Definition 5: Let $(S)_{H}^{*}$ be the set of all formal expansions:

$G(\omega)=\sum_{\beta \in I} b_{\beta} H_{\beta}(\omega)$ such that

$\|G\|_{H,-q}^{2}=\sum_{\beta \in I} \beta ! b_{\beta}^{2}(2 N)^{-q \beta}<\infty$ for some $q \in N$

The fractional white noise $W_{H}(t)$ at time $t>0$ is defined by

$W_{H}(t)=\sum_{i=1}^{\infty}\left[\int_{R} e_{i}(v) \phi(t, v) d v\right] H_{\varepsilon^{(i)}}(\omega)$

for $q>(\Gamma=1.33)$

we have,

$\left\|W_{H}(t)\right\|_{H,-q}^{2}=\sum_{i=1}^{\infty} \varepsilon^{(i)} !\left[\int_{R} e_{i}(v) \phi(t, v) d v\right]^{2} \times$

$$
(2 N)^{-q \varepsilon^{(i)}}
$$

By integrability of $W_{H}(t)$ in $(S)_{H}^{*}$ for $s \in[0, t]$ then

$$
\begin{aligned}
\int_{0}^{t} W_{H}(s) d s & =\sum_{i=1}^{\infty}\left[\int_{0}^{t}\left(\int_{R} e^{i}(v) \phi(u, v) d v\right) d u\right] H_{\varepsilon^{(i)}}(\omega) \\
& =B_{H}(t)
\end{aligned}
$$

$\mathrm{y}$ reasonable assumptions,

then $t \rightarrow B_{H}(t)$ is differentiable in

$(S)_{H}^{*} \quad$ and $\quad \frac{d B_{H}(t)}{d t}=W_{H}(t) \in(S)_{H}^{*}$

justifying fractional SDE governed by

$d X(t)=\mu X(t) d t+\sigma X(t) d B_{H}(t) \quad ; X(0)=x>0$

where $x, \mu$ and $\sigma$

are constants.

We recast the equation (31) in $(S)_{H}^{*}$ :

$\frac{d X(t)}{d t}=\mu X(t)+\sigma X(t) \circ W_{H}(t)$

$$
=\left(\mu+\sigma W_{H}(t)\right) \circ X(t)
$$

Lemma 1:

(1) $F, G \in(S)_{H}^{*} \Rightarrow F \bullet G \in(S)_{H}^{*}$

(2) $\psi, \eta \in(S)_{H} \Rightarrow \psi \bullet \eta \in(S)_{H}$

And Wick calculus, we have solution of the type:

$$
\begin{aligned}
X(t) & =x e^{\circ}\left(\mu t+\sigma \int_{0}^{t} W_{H}(s) d s\right. \\
& =x \exp ^{\circ}\left(\mu t+\sigma B_{H}(t)\right.
\end{aligned}
$$

Using the property of Hermite polynomial, if $f \in L_{\phi}^{2}(R)$, we have 


$$
\begin{gathered}
e^{*}\left(\left\langle w_{v} f\right\rangle\right)=e^{*}\left(\sum_{k}\left(f, e_{k}\right)_{\phi}\left\langle w_{s} e_{k}\right\rangle\right) \\
=e\left(\left\langle w_{s} f\right\rangle-\frac{1}{2}|f|_{\phi}^{2}\right)
\end{gathered}
$$

It follows then, that

$$
e^{\circ}(\langle w, f\rangle)=\varepsilon(f) \quad \forall f \in L_{\phi}^{2}(R)
$$

And a solution is of the form

$$
X(t)=\operatorname{xexp}\left(\sigma B_{H}(t)+\mu t-\frac{1}{2} \sigma^{2} t^{2 H}\right)
$$

\section{CONCLUSION:}

In this paper, the aim of the pension fund manager is to minimize the expected utility loss function. The solution of the derived diffusion problem associated with maximization of the return of the investment of the funds over a finite time horizon and the derivation of the time-inversion for the process which is verified to be a smooth solution of the recast problem as a fractional Brownian motion with Hurst parameter $>0.5$.

\section{REFERENCES}

[1] Boulier, J.F., Huang. S. and taillard. G. (2001), Optimal management under stochastic interest rates. The case of a protected defined contribution pension fund. Insur.: Math and Econo., 28,173C189.

[2] Deelstra, G., Grasselli, M. and Koehl, P.F. (2004), Optimal design of the guarantee for defined contribution funds. J.of Econ. Dyna. and Contr., 28, 2239C2260.

[3] Duncan, T.E., Hu, Y.Z. and Pasik-Duncan, B.(2000), Stochastic calculus for fractional Brownian motion. I, SIAM J. control optim., 38, pp 582-612.

[4] Feyel, D. and Pradelle, dela.(1996), Fractional integrals and Brownian processes. Potential analysis, 10,273-288.

[5] Hu, Y. and Oksenal, B. (2003), Fractional white noise calculus and application to finance, Infinite dimensional Anal., Quant. Probab. Rel. Topics, 6, IC32.

[6] Jianwei, G. (2013), Stochastic optimal control of DC pension fund under the fractional Brownian motion, Appl. Math. Inf. Sci. 7, No 2, 571-578.

[7] Mamadou, A.D. and Youssef, O. (2011), A linear stochastic differential equation driven by a fractional Brownian motion with Hurst parameter $>1 / 2$. Stat. \& Prob. Lett.,8, 1013-1020.

[8] Mandelbrot,B.B. and Van Ness, J.W. (1968), Fractional Brownian motions, fractional noises and applications, SIAM Review 10(4), 422-437.

[9] Merton, R. (1969), Lifetime portfolio selection under uncertainty: The continuous-time case. Review of Economics and Statistics, 51, $247 \mathrm{C} 257$.

[10] Onwukwe , I. and Bright, O.O.(2015), Optimal Plan for DC and DB pension schemes with stochastic income under the Poisson model, J. of NAMP, vol 29, pp 181-190

[11] Rogers, L.C.G. (1997), Arbitrage with fractional Brownian motion, math. Finance 7,95-105.

[12] Ruzmaikina, A.A. (2000), Stieltjes integrals of Holder continuous functions with applications to fractional Brownian mation, J. stat. phys., 100, 1049-1069.

[13] Samko, S.G., Kilbras, A.A. and Marichev, O.I. (1993), Fractional integrals and derivatives. Theory and applications. Gordon and Breach.

[14] Young, L.C. (1936), An inequality of the Holder type connected with Stieltjes integration, Acta math, 67, 251-282.

[15] Zahle , M. (1998), Integration with respect to fractal function and stochastic calculus. I. Prob. Theory relat. Fields, 111, 333-374. 